\title{
Perfil clínico-epidemiológico de las infecciones por virus respiratorios en adultos hospitalizados durante la estación de influenza 2004
}

\author{
Ricardo Rabagliati B., Michel Serri V., Cecilia Perret P., Ana M. Guzmán D., \\ Teresa Azócar A., Leila Habash A., Claudia Espinoza L. y Marcela Ferrés G.
}

\section{Clinical and epidemiological characteristics of respiratory virus infections among adults hospitalized during 2004 influenza season}

Background: During yearly influenza (FLU) season, FLU viruses are well represented among hospitalized patients as in the community. Also, other respiratory viruses could be represented among adult in-patients. Aim: to describe the presence and clinical- epidemiological characteristics of non-FLU respiratory virus infections (respiratory syncytial-RSV, parainfluenza and adenovirus-ADV) among hospitalized adults during FLU season and to compare with FLU-A (IA) or -B (IB) cases. Patients and Methods: Adult patients hospitalized at Hospital Clínico Universidad Católica between May to July 2004 with a respiratory virus infections confirmed by rapid antigen test or direct immunofluorescence of IA, IB (Flu group) or RSV, parainfluenza (1-2-3) and ADV (non-Flu group) were included. Results: 86 cases were identified: $73.5 \%$ FLU (48.2\% IA, 25.3\% IB) and 26.5\% non-FLU (15.7\% parainfluenza-2; 8.4\% RSV; 1.2\% parainfluenza-3; $1.2 \%$ ADV). No differences were observed in general characteristics and evolution of patients. In FLU-group were more frequently observed myalgia, cough, hospitalization due to febrile syndrome, higher values of $\mathrm{C}$ reactive protein and band leukocytes count $(\mathrm{p}<0.05)$. Conclusions: During 2004 FLU season a $26.5 \%$ of respiratory viral infection were due to non-FLU viruses among adult hospitalized in our centre. The difficulty to difference FLU vs. non-FLU infections, suggest that it is necessary to include other respiratory virus in the viral etiological diagnosis, even in FLU season.

Key words: Influenza virus; respiratory syncytial virus; parainfluenza virus; adenovirus; respiratory viral infection

Palabras claves: virus influenza; virus respratorio sincicial; virus parainfluenza; adenovirus; infección respiratoria viral

\section{Introducción}

$\mathrm{L}$ as infecciones por virus respiratorios constituyen un motivo frecuente de consulta médica ambulatoria $^{1,2}$. Durante los últimos años se ha logrado evidenciar la importancia de los virus respiratorios como causa de hospitalización en sujetos adultos $^{3}$, tanto por las manifestaciones del cuadro viral en particular en pacientes con patología cardiopulmonar de base, como por las consecuencias de sobreinfección bacteriana y descompensación de patologías de base, determinando incluso mortalidad en grupos de edades extremas $^{3,4}$. Nuestro grupo ha descrito por primera vez en Chile el impacto clínico y epidemiológico del reconocimiento de virus influenza-A (IA) como motivo de hospitalización en adultos con síndrome febril y/o insuficiencia respiratoria durante la temporada de in- fluenza $(\mathrm{FLU})^{5}$. Sin embargo, se debe tener en cuenta que durante los meses de otoño-invierno circulan no sólo virus IA, sino también virus influenza-B (IB), parainfluenza, virus respiratorio sincicial (VRS), adenovirus (ADV), coronavirus, rinovirus ${ }^{4,6}$, y más recientemente se ha identificado metapneumovirus en poblaciones pediátricas y de pacientes inmunocomprometidos $^{7,8}$. Existen pocos estudios que evalúen la presencia de otros virus respiratorios entre adultos durante la temporada de FLU; recientemente se publicaron en la literatura internacional interesantes datos, correspondientes al invierno 2002, en que se demuestra etiología viral en $39 \%$ de pacientes consultantes por cuadros respiratorios agudos, de los cuales el porcentaje de FLU y no FLU era de 52 versus $48 \%{ }^{9}$. En nuestro conocimiento no existen trabajos similares en Chile entre adultos hospitalizados.
Pontificia Universidad Católica de Chile, Santiago, Chile: Departamento de Medicina Interna (RRB, MSV) Laboratorios de Virología, Centro de Investigaciones Médicas (CPC, TAA, LHA, MFG)

UDA de Laboratorios Clínicos (AMGD, CEL)

Recibido: 27 septiembre 2005 Aceptado: 7 marzo 2006

Correspondencia a: Ricardo Rabagliati Borie rabagli@med.puc.cl 
La vigilancia de virus respiratorios del Laboratorio de Virología del Centro de Investigaciones Médicas de la Pontificia Universidad Católica de Chile, que incluye muestras de niños y adultos de 5 centros centinelas de la ciudad de Santiago, entrega semanalmente información actualizada del comportamiento de los virus respiratorios en la comunidad, accesible a través de Internet ${ }^{10}$. La información entregada durante los últimos años evidencia la circulación simultánea de diferentes tipos de virus respiratorios en la comunidad durante los meses de invierno. Esta observación plantea la interrogante si el estudio que se realiza al paciente con sospecha clínica de infección respiratoria viral debe incluir sólo la determinación de IA e IB o se debe considerar la búsqueda de virus no FLU (parainfluenza, VRS, ADV, rinovirus, coronavirus, etc.). El objetivo principal de este estudio fue describir la presencia y características clínico-epidemiológica de adultos hospitalizados con infección confirmada por virus respiratorios no FLU durante la temporada de FLU 2004, y como objetivo secundario establecer una comparación entre aquellos con IA o IB versus VRS, parainfluenza y ADV.

\section{Pacientes y Métodos}

Se diseñó un estudio retrospectivo que incluyó pacientes adultos, sobre 15 años de edad, con infección viral respiratoria demostrada, internados en el Hospital Clínico de la Pontificia Universidad Católica de Chile, entre el 1 de mayo y 24 de julio de 2004 (semanas epidemiológicas 17 a 29).

Los casos fueron identificados a partir de los registros del laboratorio clínico con resultado positivo de

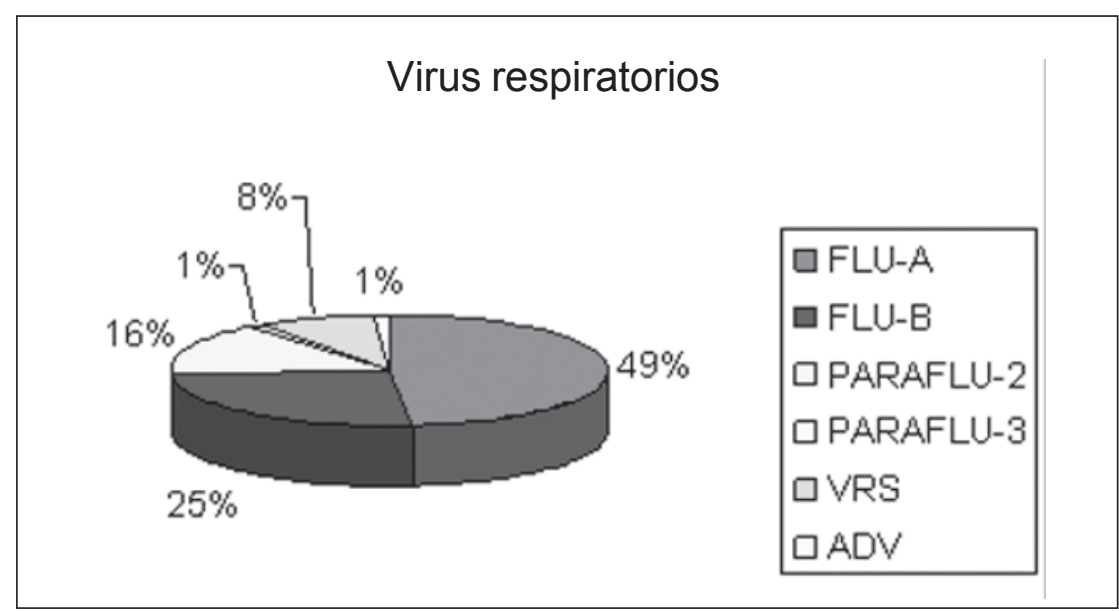

Figura 1. Distribución relativa de agentes infecciosos virales diagnósticados. FLU-A: influenza-A, FLU-B = influenza-B, ParaFLU-2 = parainfluenza- 2, ParaFLU- $3=$ parainfluenza- 3, VRS = virus respiratorio sincicial, $A D V=$ adenovirus). pruebas de diagnóstico rápido para virus IA o IB: Directigen Flu A+B (Becton, Dickinson and Company, Shannon Ireland) o inmunofluorescencia directa (IFD) para IA e IB, parainfluenza (1, 2 y 3), ADV y VRS, en muestras obtenidas por hisopado nasofaringeo.

De la ficha clínica se extrajeron los siguientes antecedentes: edad, sexo, enfermedades crónicas basales, motivo y lugar de ingreso, adquisición de la enfermedad, uso de vacuna anti-influenza y terapias antimicrobianas previas al diagnóstico, hallazgos del examen físico y laboratorio, evolución clínica y complicaciones.

Para efecto de comparación de los grupos, los pacientes fueron clasificados en dos categorías según su diagnóstico virológico. El primero incluyó a los que presentaron infección por virus IA o IB (grupo influenza, FLU) y el segundo a los que tuvieron demostrado uno de los siguientes agentes virales: VRS, parainfluenza serotipos 1, 2 o 3, o ADV (grupo noinfluenza, no-FLU).

Análisis estadístico: para el análisis estadístico de los datos recolectados y se utilizó el programa SPSS for Windows. Los valores no continuos se presentaron en porcentaje del total y las variables continuas en promedio con su desviación estándar; excepto la edad en que se presenta la media y su rango de edad correspondiente. Se utilizó el método $\mathrm{c}^{2}$, test de Fisher o test t Student para muestras no pareadas según correspondía. Se utilizó un $\mathrm{p}$ de 0,05 para establecer significancia estadística.

\section{Resultados}

Comportamiento epidemiológico de los agentes virales. Durante el período de estudio se identificaron 83 exámenes positivos para virus respiratorios correspondientes al mismo número de pacientes. Sesenta y uno $(73,5 \%)$ de los casos correspondían al grupo FLU y $22(26,5 \%)$ al grupo no- FLU. La identificación viral demostró $40(48,2 \%)$ casos de virus IA, $21(25,3 \%)$ virus IB, $13(15,7 \%)$ virus parainfluenza-2, $7(8,4 \%)$ VRS, $1(1,2 \%)$ virus parainfluenza-3 y $1(1,2 \%) \mathrm{ADV}$. (Figura 1)

Se detectó presencia de IA e IB durante las 13 semanas de observación, con un predominio de IA durante las primeras semanas, un alza máxima en la semana 21, y un descenso progresivo hasta la semana 28 (Figura 2A). El resto de los agentes virales se observó en menor proporción durante todo el periodo, detectándose los primeros casos de VRS a partir de la semana 26 y un ascenso paulatino en las siguientes semanas (Figura 2A). Al comparar la distribución de los virus con respecto a su circulación en la ciudad de 
Santiago (2B), en ambos se pudo apreciar presencia de los diferentes virus respiratorios a lo largo del periodo: predominio de IA, presencia de IB, una mayor actividad relativa de parainfluenza en el hospital, y aparición de VRS durante las últimas semanas tanto en la vigilancia comunitaria como en el hospital, siendo de mayor magnitud en el primer caso (Figuras 2A y 2B). El mayor número de VRS observado en la vigilancia está influido, entre otros factores, por la inclusión de la población pediátrica, grupo en el que fue más frecuente el diagnóstico de este agente.

Características comparativas de la población entre grupo FLU y grupo no-FLU. No se observó diferencia estadísticamente significativa en las características demográficas y presencia de patología de base entre ambos grupos (Tabla 1). En el grupo FLU las cardiopatías fueron la segunda enfermedad basal en frecuencia, seguida por las enfermedades pulmonares (EPOC o asma bronquial) mientras que en el grupo noFLU cardiopatías y diabetes mellitus fueron las más frecuentes, después de hipertensión arterial. En ambos grupos hubo sobre $10 \%$ de hospederos inmunocomprometidos (incluidos pacientes sometidos a trasplantes de órganos sólidos, infectados con VIH, quimioterapia, bajo efecto de inmunosupresores o corticoterapia crónica).

El motivo de ingreso más frecuente fue síndrome febril en los pacientes con FLU en relación a los noFLU (44,3 versus 9,1\% $\mathrm{p}=0,007)$. Aproximadamente $30 \%$ de los ingresos en ambos grupos fue por insuficiencia respiratoria y $30 \%$ de los pacientes en cada grupo requirió ingresar a una unidad de paciente crítico (intermedio o intensivo).

Siete en 61 (11\%) de las infecciones por FLU y 5/22 $(23 \%)$ de las infecciones virales no-FLU fueron adquiridas en el hospital; en 3 casos las infecciones se diagnosticaron en pacientes post-operados, representando $25 \%(3 / 12)$ de las infecciones adquiridas en forma nosocomial.

No hubo diferencias significativas en cuanto al antecedente de vacunación anti-influenza (18 versus $16 \%)$.

Manifestaciones clínicas. En el grupo FLU la temperatura $>38{ }^{\circ} \mathrm{C}$ fue más frecuentemente objetivada que en el grupo no- FLU (60,7 vs. $36,4 \%$ p = NS) (Tabla $2)$. Los síntomas de compromiso sistémico como mialgias, calofríos, así como la tos fueron también significativamente más frecuentes en el grupo FLU. En cambio la disnea fue más frecuente en el grupo noFLU (39,3 versus 63,6\% p =0,05). (Tabla 2). Las características de la expectoración ni la frecuencia de síntomas gastrointestinales fueron significativamente diferentes entre ambos grupos. Los parámetros hemodinámicos (presión arterial, frecuencia cardíaca y respi- ratoria) fueron significativamente similares entre ambos grupos.

Hallazgos de laboratorio. Los pacientes del grupo FLU presentaron mayor leucocitosis y mayor desviación izquierda (expresado en porcentaje de baciliformes $>5 \%)$ en comparación al grupo no- FLU $(p=0,02)$ (Tabla 3). La proteína $\mathrm{C}$ reactiva $(\mathrm{PCR})$ resultó más elevada en los pacientes con FLU $(6,7 \pm 5 \mathrm{mg} / \mathrm{dl}$ vs 3,7 $\pm 3 \mathrm{mg} / \mathrm{dl} ; \mathrm{p}=0,005)$. (Tabla 3 ).

Más de un tercio de los pacientes de ambos grupos estaban recibiendo tratamiento antibacteriano al momento de diagnosticarse la infección viral.

Evolución y complicaciones. El 8,1\% de los pacientes del grupo FLU y $9 \%$ de los pacientes del grupo noFLU debieron trasladarse a una unidad de mayor complejidad. En todos los pacientes de ambos grupos se adoptaron medidas para el control de infección nosocomial: aislamiento de gotitas en FLU (pieza indi-
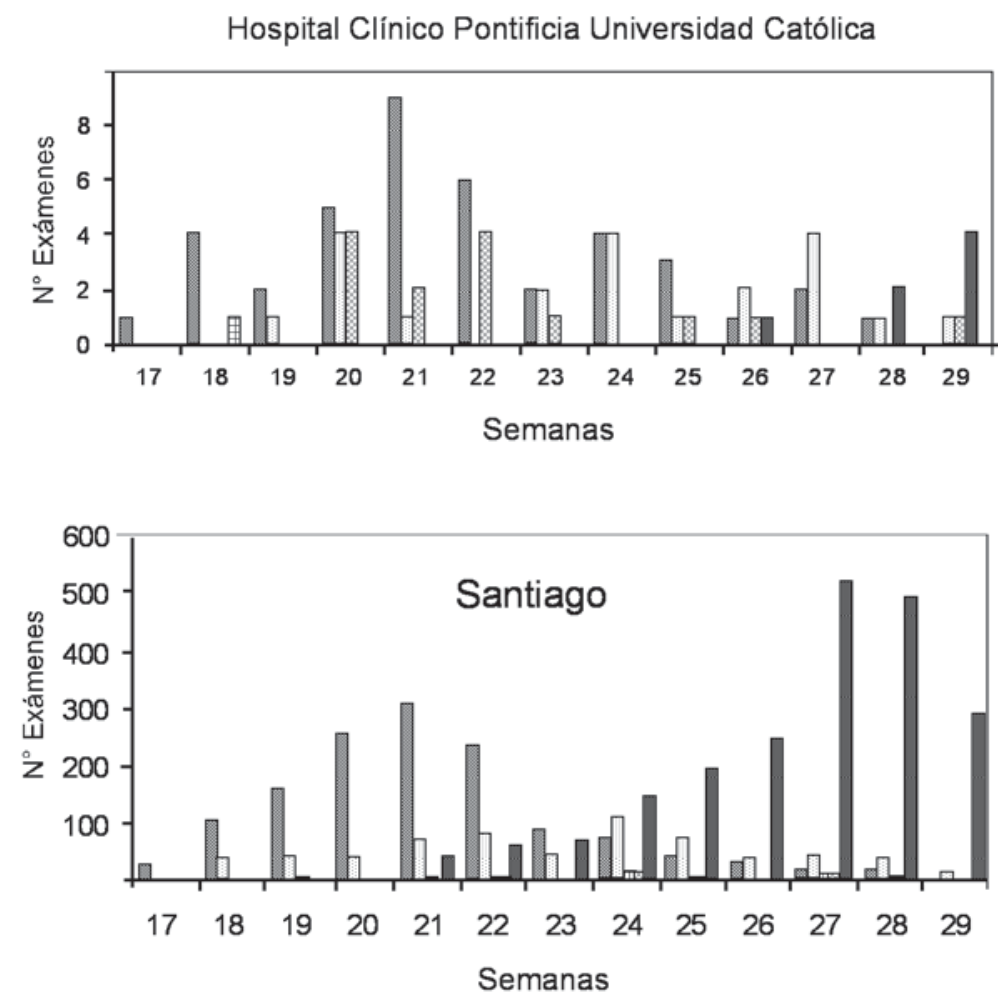

Influenza A $\square$ Influenza B 田 Adenovirus Oarainfluenza VRS

Figura 2. (2A): Circulación de los distintos virus respiratorios en el Hospital Clínico Pontificia Universidad Católica de Chile (HCUC) y (2B): la ciudad de Santiago de Chile durante las semanas epidemiológicas 17 a 29. 


\begin{tabular}{|c|c|c|c|}
\hline Antecedentes & FLU $(N=61)$ & no-FLU $(N=22)$ & $\mathbf{p}$ \\
\hline Edad media en años (rango) & $\begin{array}{c}68 \\
(16-101)\end{array}$ & $\begin{array}{c}68 \\
(17-86)\end{array}$ & NS \\
\hline Sexo masculino: femenino & $\begin{array}{c}25: 36 \\
(41: 59 \%)\end{array}$ & $\begin{array}{c}11: 11 \\
(50: 50 \%)\end{array}$ & NS \\
\hline \multicolumn{4}{|l|}{ Lugar de ingreso } \\
\hline Sala común & $41(67,2 \%)$ & $17(68 \%)$ & NS \\
\hline U. pacientes críticos & $20(32,8 \%)$ & $8(32 \%)$ & NS \\
\hline \multicolumn{4}{|l|}{ Enfermedades basales } \\
\hline Hipertensión arterial & $25(41 \%)$ & $8(36,4 \%)$ & NS \\
\hline Cardiopatías & $19(31,1 \%)$ & $5(22,7 \%)$ & NS \\
\hline Inmunosupresión\# & $8(13,1 \%)$ & $4(18,2 \%)$ & NS \\
\hline EPOC-asma bronquial & $9(14,8 \%)$ & $4(16,2 \%)$ & NS \\
\hline Diabetes mellitus & $7(11,5 \%)$ & $5(22,7 \%)$ & NS \\
\hline Vacuna anti-influenza & $11(18 \%)$ & $4(16 \%)$ & NS \\
\hline \multicolumn{4}{|l|}{ Adquisición } \\
\hline Extrahospitalaria & $54(88,5 \%)$ & $17(77,3 \%)$ & NS \\
\hline Intrahospitalaria & $7(11,5 \%)$ & $5(22,7 \%)$ & NS \\
\hline \multicolumn{4}{|l|}{ Motivo de ingreso } \\
\hline Síndrome febril & $27(44,3 \%)$ & $2(9,1 \%)$ & 0,007 \\
\hline Insuficiencia respiratoria & $18(29,5)$ & $8(32 \%)$ & NS \\
\hline \multicolumn{4}{|c|}{$\begin{array}{l}\text { \# Trasplante de órganos sólidos, quimioterapia, infección por VIH, corticostesteroides, } \\
\text { inmunosupresores. EPOC: enfermedad pulmonar obstructiva crónica } \\
\text { NS = valor p no significativo }\end{array}$} \\
\hline
\end{tabular}

Tabla 2. Comparación de características semiológicas relevantes entre los grupos influenza (FLU) y no influenza (no-FLU)

\begin{tabular}{|lrrll|} 
Signo/síntoma & FLU (n: 61) & no-FLU (n: 22) & p \\
Fiebre: & 1 & $(1,6 \%)$ & $1(4,5 \%)$ & NS \\
$\quad$ No & $23(37,7 \%)$ & $13(59,1 \%)$ & NS \\
$\quad<38{ }^{\circ} \mathrm{C}$ & $37(60,7 \%)$ & $8(36,4 \%)$ & NS \\
$>38{ }^{\circ} \mathrm{C}$ & $31(50,8 \%)$ & $6(27,3 \%)$ & 0,05 \\
Calofríos & $34(55,7 \%)$ & $6(27,3 \%)$ & 0,022 \\
Mialgias & $48(78,7 \%)$ & $17(77,3 \%)$ & NS \\
Decaimiento & $18(29,5 \%)$ & $6(27,3 \%)$ & NS \\
Cefalea & $24(39,3 \%)$ & $14(63,6 \%)$ & 0,05 \\
Disnea & $53(86,9 \%)$ & $14(63,6 \%)$ & 0,018 \\
Tos & $16(26,2 \%)$ & $3(13,6 \%)$ & NS \\
Expectoración: & $8(13,1 \%)$ & $3(13,6 \%)$ & NS \\
$\quad$ Ausente & $24(39,3 \%)$ & $6(27,3 \%)$ & NS \\
$\quad$ Mucosa & $1(1,6 \%)$ & $2(9,1 \%)$ & NS \\
$\quad$ Muco purulenta & $8(13,1 \%)$ & $2(9,1 \%)$ & NS \\
$\quad$ Purulenta & $4(6,6 \%)$ & $3(13,6 \%)$ & NS \\
Vómitos & $25(41 \%)$ & $8(32 \%)$ & NS \\
Diarrea & & & \\
Uso de antibacterianos previos & & & \\
\hline NS =valor p no significativo & & &
\end{tabular}

vidual o en cohorte) y aislamiento de contacto en noFLU.

Sólo 2 pacientes con IA fueron tratados con oseltamivir, el resto recibió amantadina, ambas en dosis ajustadas a la función renal y edad, por un tiempo promedio de $4 \pm 1$ día. Todos los pacientes con IB fueron tratados con oseltamivir, dosificación ajustada a la función renal, por un periodo promedio de $5 \pm 1$ día.

Las complicaciones fueron más frecuentes en los pacientes con FLU, a pesar de no haber diferencia significativa entre los grupos. La insuficiencia respiratoria (necesidad de oxigeno mayor al 30\%) fue la complicación más frecuente en ambos grupos (47,5 versus $36 \%$ ). Las complicaciones cardiovasculares fueron $24,5 \%$ en el grupo FLU y $18,2 \%$ en el grupo no- FLU. El compromiso de conciencia (delirium) se presentó en $16,4 \%$ de los pacientes con FLU y $9 \%$ de los con virosis no-FLU, sin relación a la terapia recibida. Sólo 3 pacientes con IA presentaron probable sobreinfección bacteriana: un caso otitis media aguda y dos casos neumonía, aunque sin precisión etiológica.

\section{Discusión}

Los resultados presentados confirman que entre los pacientes adultos hospitalizados se refleja el comportamiento de los virus respiratorios prevalentes en la comunidad.

Durante el periodo estudiado hubo predominio de IA, seguido en frecuencia de IB y luego virus no-FLU, lográndose observar la aparición de VRS de modo simultáneo a como se apreció en la comunidad. Este estudio permite demostrar que entre adultos hospitalizados en nuestro centro con infección por virus respiratorios hasta $25 \%$ presentaba un virus no-FLU (VRS, ADV y parainfluenza), durante las semanas de actividad de FLU del año 2004, lo cual reflejaba el comportamiento de los virus en la comunidad durante ese año. Al comparar la distribución de los virus en nuestros paciente hospitalizados con los datos de la ciudad de Santiago de Chile, se comprobó que circularon los mismos agentes etiológicos y con un patrón epidemiológico semejante: un primer brote de infecciones por virus IA y un segundo brote causado por VRS. Es razonable suponer que en otros centros asistenciales se haya observado el mismo fenómeno. Nos parece importante promover la identificación de los casos, para lo cual es altamente recomendable mantenerse en conocimiento de la información entregada por las redes de vigilancia disponible para agentes virales, ya que permite conocer el comportamiento de estos agentes en la población y anticiparse para tomar medidas tanto diagnósticas, de precaución para evitar su trans- 
misión nosocomial y, eventualmente, de tratamiento anti viral en los pacientes que son hospitalizados.

Una menor presencia de VRS entre los adultos hospitalizados comparados con los datos de la vigilancia en la comunidad está influido por la inclusión de niños en este segundo grupo. Existen otras explicaciones para esta diferencia, es posible plantear que VRS afecta en menor frecuencia a adultos, que el cuadro clínico sea de menor intensidad y motive menos hospitalizaciones o, que nos enfrentamos a un problema de diagnóstico etiológico, el empleo de técnicas de mayor sensibilidad podría evidenciarlo de mejor forma, tal como lo sugiere nuestra experiencia durante 2005 (datos aún no publicados).

En el estudio de JK Louie et al, efectuado en California, E.U.A. ${ }^{9}, 48 \%$ de las infecciones eran debidas a virus no FLU: VRS, parainfluenza-1, ADV, coronavirus, picornavirus y metapneumovirus, causando los 3 primeros $15 \%$ de las infecciones. Nuestro porcentaje es mayor, lo que evidencia el particular comportamiento de los virus cada año y su variabilidad en cada lugar, pero ambos trabajos confirman la presencia de otros virus respiratorios durante la temporada de FLU entre adultos. Será motivo de vigilancia durante las próximas temporadas la presencia de otros grupos de virus no incluidos en nuestro estudio, en población adulta, en particular entre aquellos hospitalizados.

En el análisis de las características clínico-epidemiológicas de ambos grupos, tanto FLU como noFLU, se pudo destacar que principalmente afectó a adultos sobre 60 años de edad y con enfermedades crónicas cardiovasculares (cardiopatías y/o hipertensión arterial), enfermedad pulmonar crónica, diabetes mellitus e inmunosupresión. Lo anterior concuerda con la literatura internacional que describe que aproximadamente $20 \%$ de los pacientes con infecciones virales no- FLU tienen una cardiopatía de base y $18 \%$ una enfermedad pulmonar crónica; en pacientes inmunocomprometidos la incidencia es entre 5 y $15 \%{ }^{11-13}$.

El antecedente de vacunación anti-influenza era de similar frecuencia en ambos grupos (18 y 16\%). En el grupo no-FLU también había un elevado número de sujetos que tenían plena indicación de recibir vacuna. Para aquellos en que fracasó la vacuna se requiere más antecedentes para comprender esa falla. Estos datos nos deben motivar a fomentar aún más la vacunación anti-FLU entre estos pacientes ya que es una medida que beneficia sin lugar a dudas a este grupo ${ }^{14-18}$.

Por ahora no existen vacunas disponibles para virus no-FLU; la vacunación anti-VRS está en plena evaluación en población pediátrica ${ }^{19,20}$, en la medida que se reconozca con mayor frecuencia este virus en adultos se podría considerar a futuro la evaluación de esta vacuna en diferentes grupos de población adulta.
Tabla 3. Comparación de los parámetros de laboratorio entre los grupos influenza (FLU) y no influenza (no-FLU)

\begin{tabular}{|lccc|}
\hline Examen & FLU (n: 61) & no-FLU (n: 22) & p \\
Hemograma: & & & \\
Hematocrito \% & $39,2 \pm 5,9$ & $37,3 \pm 5,4$ & $\mathrm{NS}$ \\
Leucocitos/mm ${ }^{3}$ & $8.901 \pm 3.600$ & $6.968 \pm 3.545$ & 0,02 \\
\% de baciliformes & $9,3 \pm 7$ & $4,6 \pm 4$ & $<0,01$ \\
\% de segmentados & $71,2 \pm 12$ & $70,5 \pm 13$ & $<0,01$ \\
\% de linfocitos & $14 \pm 10$ & $16 \pm 9$ & $\mathrm{NS}$ \\
VHS (mm/h) & $42,3 \pm 29$ & $45,9 \pm 27$ & $\mathrm{NS}$ \\
PCR (mg/dl) & $6,7 \pm 5$ & $3,67 \pm 3,1$ & 0,005 \\
Radiografía de tórax & & 14 & $\mathrm{NS}$ \\
(n) & 33 & $7(50 \%)$ & $\mathrm{NS}$ \\
Normal & $12(36,4 \%)$ & $2(14,3)$ & \\
Consolidación & $5(15,2 \%)$ & & \\
NS = valor p no significativo & & & \\
\hline
\end{tabular}

Los pacientes con FLU se comportaron clínicamente con un cuadro clínico de mayor intensidad, con mayor compromiso sistémico y sintomático que los pacientes con infecciones por virus no- FLU, lo que concuerda con la literatura científica ${ }^{21}$. Los exámenes de laboratorio también demostraban una mayor compromiso inflamatorios en FLU, expresado con mayor leucocitosis, desviación izquierda y valores de PCR. Lo anterior se correlaciona con el mayor número de traslados a unidades de pacientes críticos y una mayor incidencia de complicaciones respiratorias, cardiovasculares y neuro-siquiátricas (delirium).

La demostración virológica es muy importante ya que la identificación viral específica permite iniciar una terapia antiviral adecuada cuando corresponda, evitar el uso innecesario de antibacterianos e implementar las medidas de control de infecciones entre los pacientes hospitalizados. Para la detección de virus FLU el test rápido tiene una sensibilidad de 67 a $96 \%$ y una especificidad 88 a $97 \%$; la IFD una sensibilidad y especificidad mayor al $90 \%{ }^{22}$. Para virus no-FLU el test rápido de VRS tiene una sensibilidad y especificidad de 60 a $70 \%$ y 90 a 95\%, respectivamente ${ }^{23}$. La inmunofluorescencia tiene sensibilidad variable según el tipo de virus no-FLU: 70 a 95\% en VRS y parainfluenza, 50 a $75 \%$ para adenovirus ${ }^{24,25}$. Respecto a la elección del test a realizar, dependerá de la sospecha diagnóstica y de la vigilancia en la comunidad; este último punto es relevante ya que en años en que la circulación viral es múltiple, como el año 2004, se debería privilegiar técnicas que permitan testear varios agentes en forma simultánea, con la mayor sensibilidad y especificidad posible.

Según antecedentes disponibles en la literatura cien- 
tífica, 15 a $20 \%$ de las infecciones por FLU y VRS en pacientes internados son de origen intrahospitalario ${ }^{16,26}$ sin describirse su cuantía para el resto de las infecciones virales; en nuestra experiencia la frecuencia osciló entre 10 y $25 \%$ para ambos tipos de infecciones. Cabe destacar que, a pesar de la baja frecuencia de la infección en post-operados, estos corresponde a un cuarto de las infecciones intrahospitalarias, cifra importante de retener y tener presente al momento de evaluar pacientes quirúrgicos durante los meses invernales, como causa de fiebre post operatoria.

En conclusión, este trabajo demuestra que junto a FLU, otros virus respiratorios como VRS, parainfluenza y ADV, también están presentes entre adultos hospitalizados, en una magnitud que refleja su comportamiento en la comunidad. Su rápida identificación es importante para realizar una adecuada terapia, evitando el uso innecesario de antimicrobianos e implementar las medidas de control intrahospitalario oportunas y adecuadas para evitar brotes nosocomiales.

\section{Resumen}

El comportamiento epidemiológico del virus influenza (FLU) en la comunidad se refleja en el hospital, es planteable que la actividad comunitaria de otros virus respiratorios también se traduzca en un alza de las internaciones por estos agentes. Objetivo: describir la presencia y características clínico-epidemiológicas de infecciones por virus respiratorios no-FLU (virus respiratorio sincicial-VRS, parainfluenza y adenovirusADV) entre adultos hospitalizados durante la temporada de influenza y establecer una comparación con virus influenza-A (IA) o -B (IB). Pacientes y Método: Adultos internados en Hospital Clínico Universidad Católica, de mayo a julio de 2004, con infección por IA o IB, y VRS, parainfluenza (1-2-3) o ADV demostrada por test rápido o inmunofluorescencia directa. Resultados: Se identificaron 86 casos: $73,5 \%$ FLU (48,2\%, IA y $25,3 \%$ IB) y $26,5 \%$ no-FLU. (15,7\% parainfluenza2; $8,4 \%$ VRS, $1,2 \%$ parainfluenza-3 y $1,2 \%$ ADV). El grupo con FLU presentó más frecuentemente mialgias, tos, hospitalización por síndrome febril, mayores valores de PCR y porcentaje de baciliformes $(\mathrm{p}<0,05)$. Conclusiones: Durante la temporada de influenza 2004, $26,5 \%$ de las infecciones entre adultos hospitalizados fueron causadas por virus no-FLU. La dificultad en diferenciar infecciones por virus FLU de no-FLU, plantea la necesidad de ampliar el estudio de la etiología viral incluso durante la temporada de FLU.

\section{Referencias}

1.- Nicholson K, Wood J, Zambon M. Influenza. Lancet 2003; 362: 1733-45.

2.- Simonsen L, Clarke M, Williamson D, Stroup D, Arden N, Schonberger L. The impact of influenza epidemics on mortality: Introducing a severity index. Am J Public Health 1997; 87: 1944-50.

3.- Thompson W W, Shay D K, Weintraub E, Brammer L, Cox N, Anderson L J. Mortality associated with influenza and respiratory syncytial virus in the United States. JAMA 2003; 289: 179-86.

4.- Neuzil K, Maynard C, Griffin M, Heagerty P. Winter respiratory viruses and health care use: a population-based study in the Norhwest United States. Clin Infect Dis 2003: 37: 201-7.

5.- Rabagliati R, Benítez R, Fernández A, Gaete P, Guzmán AM, García P, et al. Reconocimiento de influenza-A como etiología de síndrome febril e insuficiencia respiratoria en adultos hospitalizados durante brote en la comunidad. Rev Méd Chile 2004; 132: 317-24.

6.- Breese C. Respiratory syncytial virus and parainfluenza virus. N Engl J Med 2001; 344: 1917-28.
7.- Harmelin M E, Abed Y, Boivin G. Human metapneumovirus: a new player among respiratory viruses. Clin Infect Dis 2004; 38: 983-90.

8.- Kahn J. Human metapneumovirus: a newly emerging respiratory pathogen. Curr Opin Infect Dis 2003; 16: 255-8.

9.- Louie J K, Hacker J K, Gonzales R, Mark J, Maselli J H, Yagi S, et al. Characterization of viral agents causing acute respiratory infection in a San Francisco University Medical Center Clinic during the influenza season. Clin Infect Dis 2005; 41: 822-8.

10.- Proyecto Vigilancia Virus Respiratorios. Laboratorio de Infectología y Biología Molecular. Programa de Enfermedades Infecciosas. Pontificia Universidad Católica de Chile. Facultad de Medicina. Centro de Investigaciones Médicas. «http:// contacto.med.puc.cl/virus/virus_respiratorios /bienvenida.html»

11.- Falsey A R, Cunningham C K, Barker W H, Kouides R W, Yuen J B, Menegus M, et al. Respiratory syncytial virus and influenza A infections in the hospitalized elderly. J Infect Dis 1995; 172: 389-94.

12.- Walsh E E, Falsey A R, Hennessey P A. Respiratory syncytial and other virus infection in persons with chronic cardiopulmonary disease. Am J Respir Crit Care Med 1999; 160: 791-5.

13.- Breese C, Long C, Schnabel K. Respiratory syncytial virus infections in previously healthy working adults. Clin Infect Dis 2001; 33: 792-6.

14.- Wood A. Prevention and treatment of influenza. N Engl J Med 2000; 343: 177887.

15.- Nichol K L, Margolis K L, Wuorenma J, Von Sternberg T. The efficacy and cost effectiveness of vaccination against influenza among elderly persons living in the community. N Engl J Med 1994; 331: 778 84.

16.- Carman W F, Elder A G, Wallace L A, McAulay K, Walker A, Murray G D, et al. Effects of influenza vaccination of health care workers on mortality of elderly people in long term care: a randomized controlled trial. Lancet 2000; 355: 93-7.

17.- Gross P, Hermogenes A, Sacks H, Lau J, Levandowski R. The efficacy of influenza vaccine in elderly persons. Ann Inter Med 1995; 123: 518-27.

18.- Lee P, Matchar D, Clements D, Huber J, Hamilton J, Peterson E. Economic analysis of influenza vaccination and antiviral treatment for healthy working adults. Ann 
Intern Med 2002; 137: 225-31

19.- Greenberg H, Piedra P. Immunization against viral respiratory disease: a review. Pediatr Infect Dis J. 2004; 23: S254-61.

20.- Karron R, Wright P, Belshe R, Thumar B, Casey R, Newman F, et al. Identification of a recombinant live attenuated respiratory syncytial virus vaccine candidate that is highly attenuated in infant. J Infect Dis 2005; 191: 1093-104.

21.- Ebell M, White L, Casault T. A systematic review of the history and physical examination to diagnose influenza. J Am
Board Fam Pract 2004; 17: 1-5.

22.- Chan K, Maldeis N, Pope W, Yup A, Ozinskas A, Gill J, et al. Evaluation of the Directigen FluA+B test for rapid diagnosis of influenza virus type $\mathrm{A}$ and $\mathrm{B}$ infections. $\mathrm{J}$ Clin Microbiol 2002; 40: 1675-80.

23.- Abels S, Nadal D, Stroehle A, Bossart W. Reliable detection of respiratory syncytial virus infection in children for adequate hospital infection control management. J Clin Microbiol 2001; 39: 3135-9.

24.- Larrañaga C, Avendaño L F, Gaggero A, Suárez M, Montaldo G, Palomino A, et al.
Diagnóstico de infección por adenovirus y virus respiratorio sincicial en lactantes: Comparación entre aislamiento e inmunofluorescencia indirecta. Rev Chil Infectol 1990; 7: 167-71.

25.- Raty R, Kleemola M, Melen K, Stenvik M, Julkunen I. Efficacy of PCR and other diagnostic methods for the detection of respiratory adenoviral infections. J Med Virol 1999; 59: 66-72.

26.- Breese C. Nosocomial respiratory syncytial virus infections: The "Cold Ward" has not ended. Clin Infect Dis 2000; 31: 590-6. 